\title{
Central Amygdala Lesions Block Ultrasonic Vocalization and Freezing as Conditional But Not Unconditional Responses
}

\author{
June-Seek Choi and Thomas H. Brown \\ Department of Psychology, Yale University, New Haven, Connecticut 06520
}

\begin{abstract}
Bilateral amygdala (AM) lesions prevent the acquisition of fear-related conditional responses (CRs) in rats, a result that is most commonly concluded to reflect a learning or memory deficit. An alternative hypothesis is that AM-lesioned animals fail to acquire certain fear CRs simply because they cannot perform these behaviors. This performance-deficit hypothesis is usually invoked in regard to studies in which the $\mathrm{CR}$ is freezing, the most commonly measured behavior. Here we explore this interpretation by measuring two different behaviors [freezing and $22 \mathrm{kHz}$ ultrasonic vocalization (USV)] elicited under three conditions (during context conditioning, during subsequent retention testing, and after ejaculation) in experimental rats [that received electrolytic lesions of the central nucleus of the amygdala (ACe)] and control animals (that received a sham operation). If ACe damage produces a discrete motor deficit that specifically renders the animal unable to remain immobile, then freezing should be blocked or impaired when elicited under all three conditions, whereas USV should be spared. Alternatively, if ACe damage selectively interferes with CR formation, maintenance, or expression, then both freezing and USV should be blocked or impaired when elicited as CRs during acquisition and retention testing but spared when evoked as unconditional responses (URs) to ejaculation. ACe damage blocked or severely impaired both freezing and USV elicited as CRs but had no effect on either behavior elicited as URs. We reject the motor-deficit hypothesis and discuss some viable alternatives.
\end{abstract}

Key words: ultrasonic vocalization; freezing; amygdala; fear; ejaculation; context conditioning

\section{Introduction}

Conditional fear responses can be acquired when an aversive unconditional stimulus (US) is paired with a conditional stimulus (CS), which can be a discrete cue or a context. After one or more CS-US pairings, the CS alone may come to elicit a set of conditional responses (CRs) that reflect species-typical defensive behaviors (Blanchard and Blanchard, 1969, 1972; Kim et al., 1993). An accumulating body of evidence suggests that circuitry within the amygdala (AM) or a closely related structure is necessary for some aspect of the formation, maintenance, or expression of these CRs (Fanselow and LeDoux, 1999; Maren, 1999; LeDoux, 2000; Lee et al., 2000; Choi et al., 2001).

In addition to receiving both CS- and US-related information, the amygdala sends projections, via its central nucleus (ACe), to motor regions that are directly responsible for organizing the production of many defensive CRs (Kapp et al., 1979; LeDoux et al., 1988; Canli and Brown, 1996; LeDoux, 2000). In one neurophysiological model (Faulkner et al., 1997; Tieu et al., 1999; McGann and Brown, 2000) that captures several aspects of current thinking (Fanselow and LeDoux, 1999; Blair et al., 2001), CS-US pairings create CS-CR pathways by inducing associative long-

Received April 16, 2003; revised July 23, 2003; accepted Aug. 6, 2003.

This work was supported by National Institutes of Health Grant MH58405-05. We thank Jeffrey Blaustein for advice on the mating protocol, Sharon Furtak and Diana Padlubnaya for comments on the final manuscript, and Michael Fanselow for useful discussion.

Correspondence should be addressed to Thomas H. Brown, Department of Psychology, Yale University, 2 Hillhouse Avenue, New Haven, CT 06520-8205. E-mail: thomas.brown@yale.edu.

J.-S. Choi's present address: Center for Neural Science, New York University, 4 Washington Place, Room 809, New York, NY 10012. E-mail:choi@ens.nyu.edu.

Copyright $\odot 2003$ Society for Neuroscience $\quad$ 0270-6474/03/238713-09\$15.00/0 term potentiation (Levy and Steward, 1979; Barrionuevo and Brown, 1983; Kelso and Brown, 1986; Kelso et al., 1986) in the amygdala (for review, see Brown and Lindquist, 2003).

The question of whether "fear memories" are actually stored within the amygdala has been debated extensively. Several investigators have presented data and arguments challenging all claims that the amygdala contains the essential circuitry that encodes fear memories (Cahill et al., 1999, 2001). One source of controversy centers around the interpretation of amygdala lesion effects on freezing, the most commonly studied Pavlovian CR to aversive conditioning. According to what could be termed the motordeficit hypothesis, the reason that amygdala lesions (particularly lesions of ACe) block freezing as a CR is not because they disrupt the essential memory circuits for fear.

Instead, the deficit is proposed to result from the simple fact that the rats are physically unable to execute this particular behavior (Cahill et al., 1999, 2001). This motor-deficit hypothesis can explain certain task-related differences in the literature regarding the effect of amygdala lesions on aversive conditioning. In particular, AM lesions can block freezing as a Pavlovian CR but spare passive avoidance behavior, an instrumental response to aversive conditioning (LeDoux et al., 1990; Kim et al., 1993; Vazdarjanova and McGaugh, 1998; Cahill et al., 1999; Vazdarjanova et al., 2000).

Here we explore the motor-deficit hypothesis by measuring two different behaviors [freezing and $22 \mathrm{kHz}$ ultrasonic vocalization (USV)] elicited under three conditions (during context conditioning, during subsequent retention testing, and after ejaculation). If ACe damage produces a specific motor deficit that renders the animal unable to remain immobile, then freezing, but 
not USV, should be blocked or impaired when elicited under all three conditions. In contrast, if the damage specifically interferes with the formation, maintenance, or expression of CS-CR connections, then both freezing and USV should be blocked or impaired when elicited during acquisition and retention testing but not after ejaculation.

\section{Materials and Methods}

Design and analysis. Freezing and $22 \mathrm{kHz}$ USV have been most commonly studied as defensive reactions to threatening stimuli (Anderson, 1954; Blanchard and Blanchard, 1969; Blanchard et al., 1991; Brudzynski and Barnabi, 1993; Antoniadis and McDonald, 1999). The present experimental design makes use of the fact that both responses also occur reliably after ejaculation (Barfield and Geyer, 1972; Barfield, 1975; Van der Poel and Miczek, 1991) coupled with our preliminary findings that ejaculation-elicited freezing and USV are indistinguishable from CSelicited freezing and USV at the level of analysis performed here (our unpublished observations).

Sham-operated control and ACe-lesioned rats were compared, in terms of mean levels of responding, in each of the three eliciting conditions: during acquisition, after ejaculation, and during retention testing. A subset of the animals that underwent context conditioning after surgery were also tested for ejaculation-elicited freezing and USV before and after one-half of them received ACe lesions and the remainder received a sham operation. To help illuminate the critical circuitry, we also examined the correlation matrix for levels of responding across the eliciting conditions.

Subjects. Data were collected from 34 experimentally and sexually naive male Sprague Dawley rats (Charles River Laboratories, Kingston, NY) weighing $\sim 350 \mathrm{gm}$ at the time of surgery. Fourteen of these animals additionally participated in the mating experiment, which also used 12 ovariectomized female rats. All rats were individually housed on a $12 \mathrm{hr}$ light/dark cycle (light on at 7:00 A.M., lights off at 7:00 P.M.) with ad libitum access to food and water. Fear conditioning and testing sessions were performed during the light cycle. Mating tests were performed during the first and the second quarter of the night cycle. Animals were handled daily for $\sim 30 \mathrm{sec} / \mathrm{d}$ for a minimum of $3 \mathrm{~d}$ before the experiment and also after the surgery. All of the following procedures, including surgery and postoperative care, were in strict compliance with the Yale Animal Resource Center guidelines.

Surgery. Subjects were anesthetized with ketamine $(90 \mathrm{mg} / \mathrm{kg}, \mathrm{i} . \mathrm{m}$. and xylazine $(10 \mathrm{mg} / \mathrm{kg}$, i.m.). Petroleum-based ophthalmic ointment was applied to both eyes to prevent the cornea from drying. Body temperature was maintained by a heating pad (Braintree Scientific, Braintree, MA) for the duration of surgery. All surgical procedures were performed under aseptic conditions. Eighteen of the 34 rats, including seven that were also used in the mating test, received bilateral ACe lesions. Two lesions were made in each hemisphere using the following stereotaxic coordinates: -1.5 anteroposterior, 4.3 mediolateral, 8.2 dorsoventral; and -2.4 anteroposterior, 4.3 mediolateral, 8.5 dorsoventral (from bregma and the top of the skull for each side). The lesions were made by passing 1.0 mA anodal DC (DC LM5A lesion maker; Grass Instruments, West Warwick, RI) for 12 sec through epoxy-coated insect pin (\#00) with $0.5 \mathrm{~mm}$ of the tip exposed. The remaining 16 animals, including seven that participated in the mating test, received a sham operation in which the lesion electrode was advanced to $1 \mathrm{~mm}$ above the target structure but no current was passed.

Apparatus and measurements. Fear conditioning and testing were done in a chamber (modified from rat test cage; Coulbourn Instruments, Allentown, PA) with inside dimensions of $28 \times 25 \times 50 \mathrm{~cm}$ (length $\times$ width $\times$ height). The conditioning chamber was contained within a sound-attenuating cubicle with inside dimensions of $90 \times 60 \times 90 \mathrm{~cm}$. A regulated grid shocker (Coulbourn Instruments) was connected to the grid floor of the conditioning chamber to deliver the US. During the US, each of the eight grid bars was activated at $15 \mathrm{~Hz}$ by $8 \mathrm{msec}$ current steps supplied by the constant-current source. The grid current, conventionally measured (Choi et al., 2001), was $1.0 \mathrm{~mA}$. Mating tests were done in a different Plexiglas cage measuring $45 \times 25 \times 20 \mathrm{~cm}$. The inside of the

\section{A. Conditional USV}

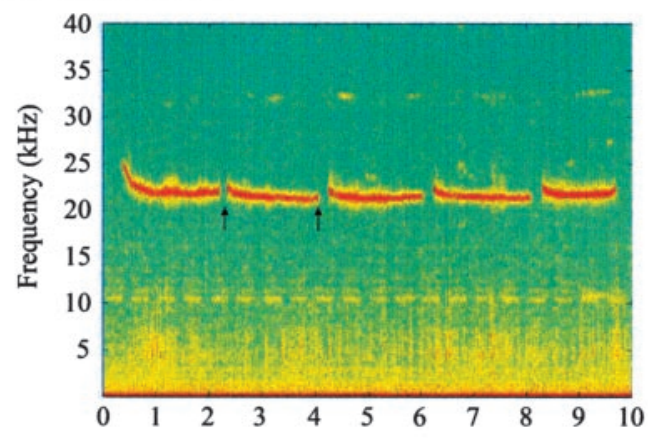

\section{B. Post-ejaculatory USV}

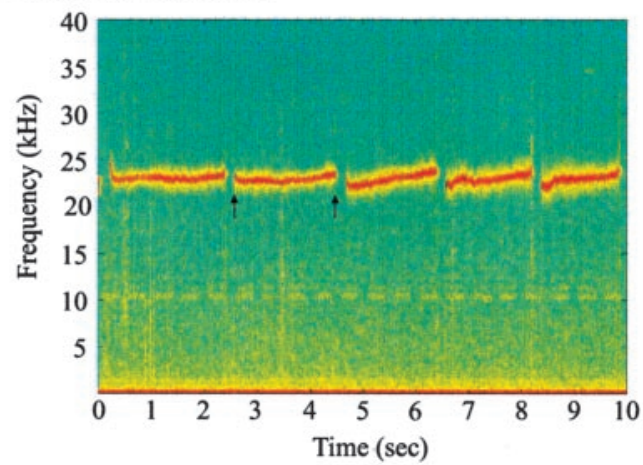

Figure 1. Spectrograms of $22 \mathrm{kHz}$ USVs elicited in control animals by mating and fear. Arrows demark an individual call in a series. The " $22 \mathrm{kHz}$ USVs" occur at $20-30 \mathrm{kHz}$, they have a narrow bandwidth of $6 \mathrm{kHz}$ or less, and they have been proposed to reflect a "negative affective state" (Brudzynski, 2001). A, Context-elicited 22 kHz USVs, which could be termed a "conditional alarm signal." $B$, Mating-elicited 22 kHz USVs, sometimes called the "post-ejaculatory song."

sound-attenuating cubicle was unlit ( $<1$ lux) during fear conditioning and dimly lit by a red light bulb during the mating test. Both were viewed in real time and recorded via an infrared video camera (CB-21; Circuit Specialists, Mesa, AZ). The complete experimental session was recorded on videocassette recording tape throughout training and testing and then replayed to assess freezing and USV.

A heterodyne bat detector (Mini-2; tuned at $22 \mathrm{kHz}$; Ultrasound Advice, London, UK), positioned inside the sound-attenuating cubicle, converted $22 \mathrm{kHz}$ USV s into audible sound. The timing of the footshock US was controlled by a programmable pulse generator (Master-8; A.M.P.I., Jerusalem, Israel). For offline analysis, a videocassette recorder recorded the video images, the audio output from the bat detector, a timing pulse that marks the beginning and the end of each conditioning session, and the timing of the grid current. In some cases, a highfrequency condenser microphone (model 7012; ACO Pacific, Belmond, CA) was placed inside the conditioning chamber to capture the original USV waveforms. The output of the microphone was amplified by a MA2 preamplifier (Tucker-Davis Technologies, Gainesville, FL) and then digitized (RP2.1 digital signal processor; Tucker-Davis Technologies) at 100 $\mathrm{kHz}$. The RP2.1 transferred data to a personal computer running Matlab (MathWorks, Natick, MA), which was also used to analyze USVs.

Figure 1 shows sonograms for USV calls that were induced by the CS (the training context) (Fig. $1 A$ ) and the US (ejaculation) (Fig. $1 B$ ) and recorded using the digital sound processor. Each call in a series is distinctively separated by a brief pause, typically of $100-200 \mathrm{msec}$. The interval between the pairs of arrows in Figure 1 is the call duration. Because the present study was not concerned with fine details of the USVs, most of the analysis used the audio output from the bat detector, which is conventional and convenient for this purpose (Blanchard et al., 1991; Antoniadis and McDonald, 1999, 2000). The output from the bat detector was passed to an operational amplifier circuit that detected the onset and 

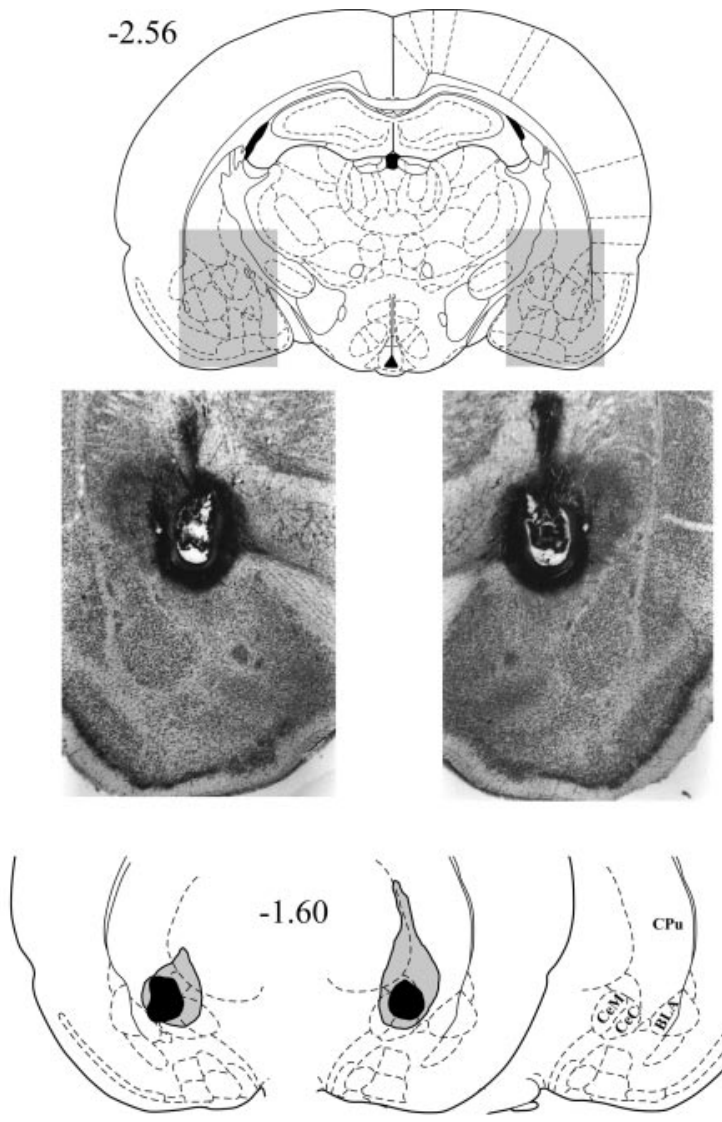

$-1.60$
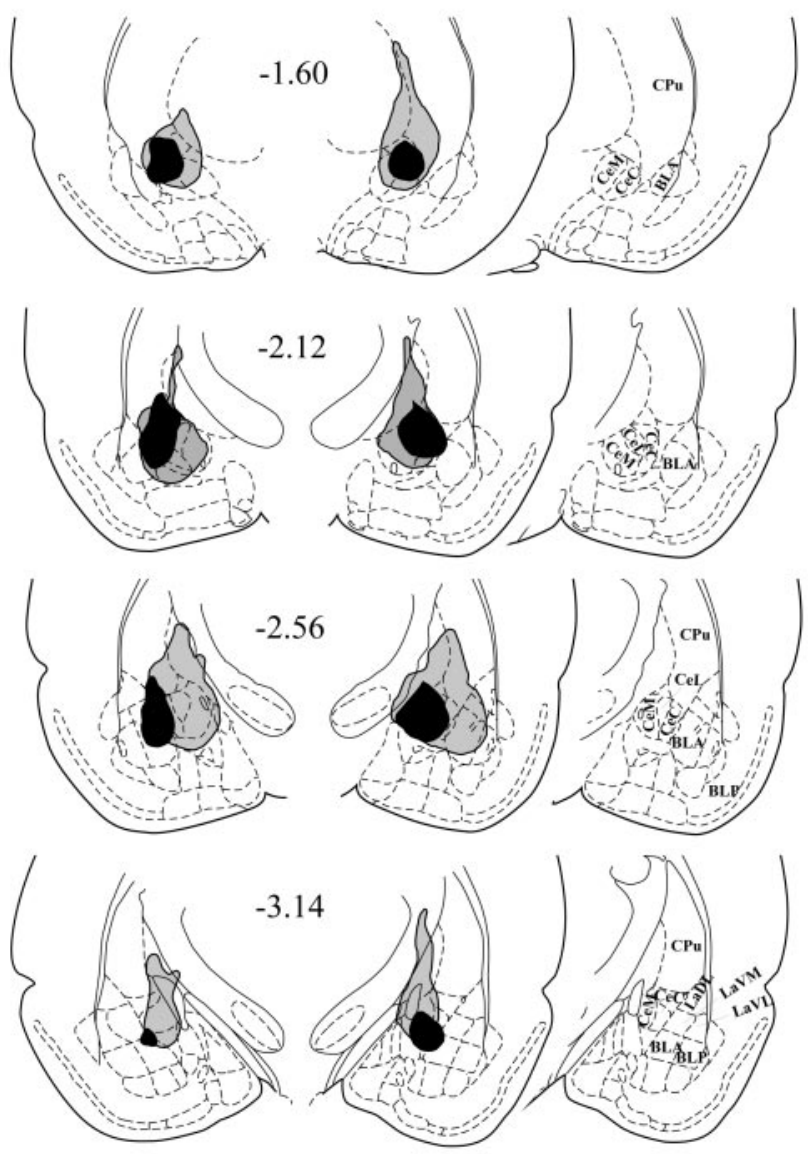

Figure 2. Histological reconstruction of lesion sites. Numbers represent distance in millimeters posterior to the bregma. Gray regions indicate the largest lesion, and the black regions indicate the smallest lesion on a given section. The plates and labeling of ACe subdivisions are from the atlas of Paxinos and Watson (1998). The capsular, medial, and lateral subdivisions of the amygdala are collectively referred to as ACe throughout text. BLA, Anterior basolateral amygdaloid nucleus; BLP, posterior basolateral amygdaloid nucleus; BLV, ventral basolateral amygdaloid nucleus; BMA, anterior basomedial amygdaloid nucleus; $\mathrm{CeC}$, capsular division of central nucleus of amygdala; CeL, lateral division of central nucleus of amygdala; CeM, medial division of central nucleus of amygdala; $\mathrm{CPu}$, caudate putamen; DEn, dorsal endopiriform nucleus; LaDL, dorsolateral lateral amygdaloid nucleus; LaVM, ventromedial lateral amygdaloid nucleus; LaVL, ventrolateral lateral amygdaloid nucleus. offset of each call using a predetermined threshold that served as an amplitude filter.

Freezing was measured identically during conditioning trials, context testing, and ejaculation. In each case, an observer pressed a button at the onset of freezing (immobility) and released it at the termination. Button presses and amplitude-filtered USV signals were simultaneously digitized at $100 \mathrm{~Hz}$, and the USV data were then further filtered. Freezing was defined conventionally and required that the rat cease movement for at least 3 sec to exclude momentary pauses in activity (Lee and Kim, 1998). To be scored as a call, the signal had to have a minimum duration of 80 msec and a minimum pause of $50 \mathrm{msec}$ separating it from preceding and following calls. The purpose of this filtering was to eliminate transient noises caused by animal's movement.

Fear conditioning procedures. The conditioning experiment, for both sham-operated control and ACe-lesioned rats, consisted of $1 \mathrm{~d}$ of context conditioning, followed by $2 \mathrm{~d}$ of testing. Baseline freezing and USV to the conditioning chamber was measured for $4 \mathrm{~min}$ before conditioning and during the 4 min of conditioning, which consisted of five footshocks (1.0 $\mathrm{mA}, 1 \mathrm{sec})$ at $1 \mathrm{~min}$ intervals. Each animal was removed from the conditioning chamber immediately after the fifth shock and then returned to its home cage. Freezing and USV were therefore only measured after the first four shocks. On each of the subsequent $2 \mathrm{~d}$ of retention testing, animals were returned to the conditioning chamber for $8 \mathrm{~min}$, during which time freezing and USV in response to this context were remeasured.

Preparation for mating. Sexual responsiveness was induced in female rats using a standard protocol (Blaustein and Wade, 1977). Ovariectomized female rats were primed by injecting estradiol ( $\beta$-estradiol 3-benzoate; $10 \mu \mathrm{g} / 0.1 \mathrm{ml}$ sesame oil, s.c; Sigma, St. Louis, $\mathrm{MO}$ ) and progesterone ( $10 \mu \mathrm{g} / 0.1 \mathrm{ml}$ sesame oil, s.c.; Sigma) $46-54$ and $4-6 \mathrm{hr}$, respectively, before the mating test. The female rats were used only once for the mating test after each priming and were then given 1-2 d without any drugs before another cycle of priming and testing was initiated.

Mating procedure. Mating tests were performed twice on 14 subjects, $3 \mathrm{~d}$ before and 1 week after surgery (ACe lesion or sham operation). Seven of these animals received ACe lesions, and seven served as shamoperated controls. In each mating test, which occurred during the first and the second quarter of the night cycle, a female rat was first acclimated to the mating chamber for $10 \mathrm{~min}$, and then a male was introduced. Mating sessions lasted $45 \mathrm{~min}$ or until the male rat engaged in an ejaculation, whichever came first. Animals were never paired with the same partner more than once.

A successful ejaculation was characterized by mounting, intromission, and vigorous pelvic thrusting, followed by post-ejaculatory USVs. Postejaculatory USVs by male rats were accompanied by a characteristic breathing pattern (Barfield and Geyer, 1972) that gave visual confirmation that the source of USVs was the male rather than the female rat. Male rats were given up to three mating opportunities both before and after the surgery. The end of the post-ejaculatory USV period was defined as a prolonged pause $(>1 \mathrm{~min})$ in vocalization and an attempt to resume mounting, at which point the rats were removed from the test chamber.

Histology. At the completion of all behavioral experiments, animals were deeply anesthetized with Nembutal, followed by halothane. They were next perfused with PBS, followed by $10 \%$ Formalin in PBS. The brain was removed from the skull, stored in $10 \%$ Formalin for a minimum of 1-2 d, transferred to a $30 \%$ sucrose solution for 3-4 d, and sectioned at $60 \mu \mathrm{m}$ with a freezing-stage microtome. Sections were mounted on gelatin-coated slides, reacted using Prussian Blue (1\% potassium ferrocyanide- $7.4 \%$ hydrochloric acid), and counterstained with cresyl violet dye. Each section was examined microscopically, and the full extent of each ACe lesion was reconstructed using a camera lucida (Axioscope with $2.5 \times$ objective; Zeiss, Oberkochen, Germany) onto anatomically matched sections from the brain atlas of Paxinos and Watson (1998). Photomicrographs were taken using the Zeiss Axiophot module and $2.5 \times$ objective.

\section{Results}

\section{Histology}

The 18 lesioned animals incurred substantial damage to ACe and partial damage to surrounding structures. Figure 2 shows a pho- 


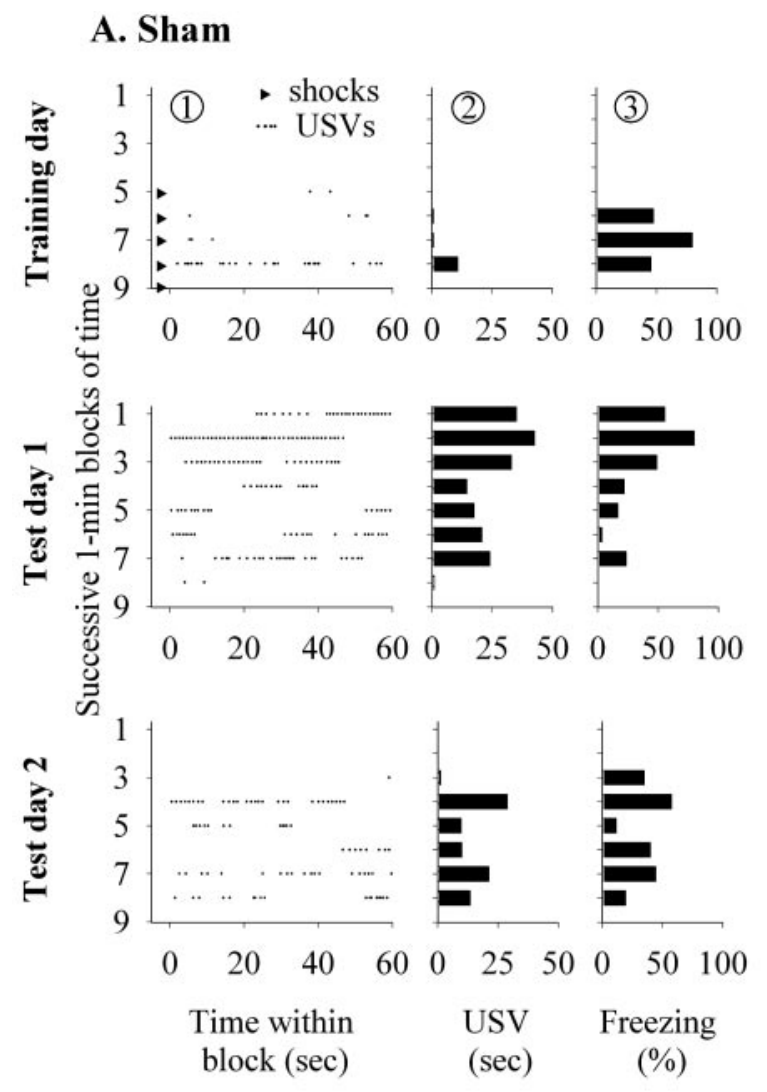

\section{B. Lesion}
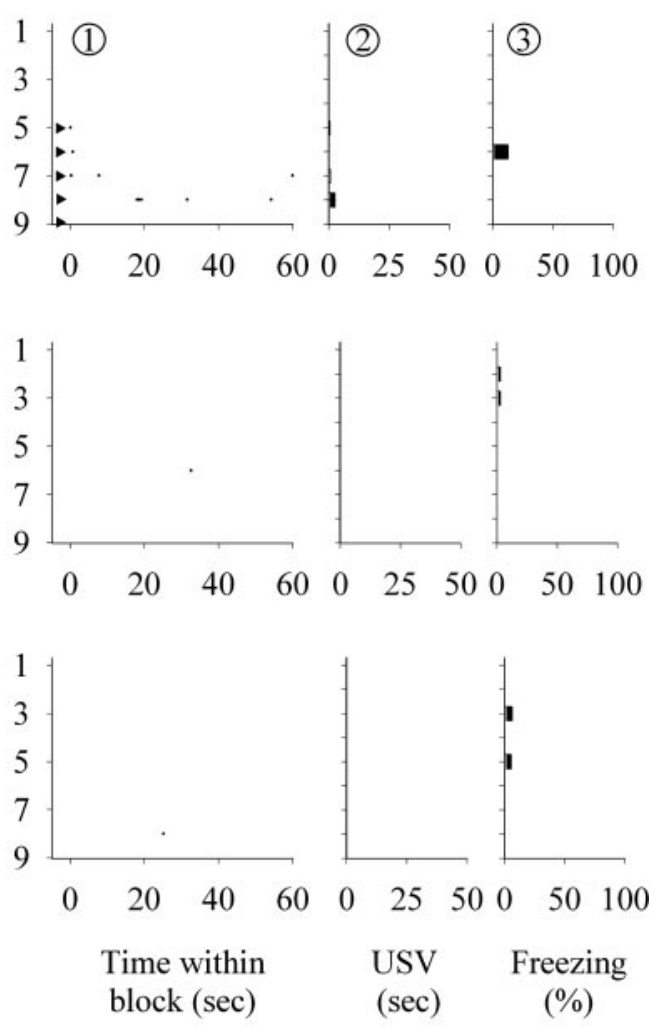

Figure 3. Typical USV and freezing patterns from two animals during conditioning (top row) and during $2 \mathrm{~d}$ of retention testing (middle and bottom rows). $A$, USV and freezing in a representative sham-operated control animal. Column 1 is an even raster plot of USVs, in which each dot marks the time of occurrence of a call. Horizontal lines are successive 1 min blocks of time. The solid triangles mark the time of successive shocks. Column 2 is a bar graph showing the total USV duration during each successive 1 min block of time. Column 3 is a bar graph showing the percentage of time that the animal was freezing in corresponding $1 \mathrm{~min}$ blocks of time. Note that the animal was unresponsive during the 4 min baseline period (rows $1-4$ ) before the first shock. $B$, USV and freezing in a representative ACe-lesioned animal. The arrangement of rows, columns, and symbols are unchanged. The almost completely empty raster plots and bar graphs dramatically illustrate the lack of responding.

tomicrograph of bilateral ACe lesions together with histological reconstructions of the smallest and the largest lesions in the group data. None of the rats was excluded from the data analysis on the basis of histological results.

\section{ACe lesion effects on USV and freezing during training and retention testing}

All 34 rats exhibited negligible freezing or USV during the $4 \mathrm{~min}$ baseline period before the onset of context conditioning. The lesion effect size was so large that it was obvious in individual animals. Figure 3 shows USV and freezing in a representative sham-operated control and ACe-lesioned rat during the pretraining period, during context conditioning, and in the two retention-testing sessions. Event raster plots (Fig. 3, two columns labeled 1) show the time course of USV in the control (Fig. 3A) and ACe-lesioned (Fig. $3 B$ ) animals during the pretraining period (lines $1-4$ on the top raster plot), during training (lines 5-8 of the top raster plot), and during the $2 \mathrm{~d}$ of testing (lines $1-8$ of the two bottom raster plots), in which each dot represents a USV call during the sample period. During periods of "continuous vocalization," the pauses between successive calls were typically less than a few hundred milliseconds (Fig. 1).

The level of USV was negligible during the pretraining period (Fig. 3, column 1, lines 1-4). The control animal vocalized throughout much of the context-testing period (Fig. $3 A$, lines 1- 8 of both test days), in contrast to the ACe-lesioned rat, whose response rate was negligible (Fig. $3 B$, lines $1-8$ of both test days).
The data were binned into successive 1 min blocks of time and plotted as histograms (two columns labeled 2) that summarize the temporal distribution of USV durations. The histograms show the level of responding during the $4 \mathrm{~min}$ pretraining period (Fig. $3 A, B$, bars corresponding to lines $1-4$ of the raster plot), the 4 min of context conditioning (bars corresponding to lines 5-8), and the two retention tests (lines $1-8$ of test days 1 and 2). The ACe-lesioned animal failed to vocalize both during conditioning and context testing.

The temporal distribution of concomitant freezing during these same time periods is plotted in the adjacent histograms (Fig. 3 , columns labeled 3), which express freezing as a percentage of the maximum possible duration. The level of freezing was negligible in both animals during the 4 min pretraining period (corresponding to lines $1-4$ of the raster plot). In contrast to the control animal (Fig. 3A), the ACe-lesioned rat (Fig. 3B) showed almost no freezing during either day of testing.

The results from these two animals are entirely representative of the group data (Fig. 4), with one exception. Whereas this particular control rat vocalized considerably more during day 1 of retention testing than during training, in the group data (Fig. 4), the mean \pm SE rates of responding were very similar during training $(28.6 \pm 4.0 \%)$ and on the first day of testing $(26.4 \pm$ $5.4 \%$ ), small differences that were not significant (Wilcoxon signed rank test; $p=0.18$ ). The lesion effect was so extreme, virtually eliminating all responding in most animals (Fig. 4), that nonparametric inferential statistics were sometimes required be- 

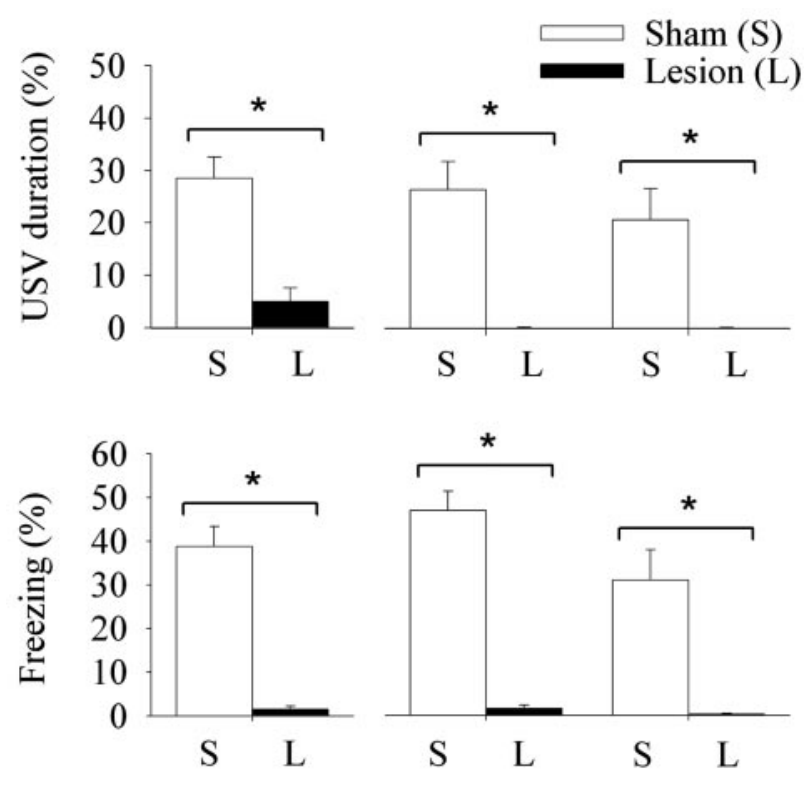

Training Test day 1 Test day 2

Figure 4. Lesion effects on freezing and USV during training and retention testing. Mean \pm SE percentage of time spent freezing and vocalizing in sham-operated animals ( $S$; white bars) and ACe-lesioned rats ( $L$; black bars). Percentages for the training session were computed on the basis of data collected during the 4 min period between the first and fifth shock. Percentages for testing were computed on the basis of the entire 8 min session. Responding was almost completely abolished in the lesioned rats ( ${ }^{*} p<0.001$ for the designated comparison). Among the sham-operated control animals, the levels of freezing and vocalization were similar during training and the first day of retention testing. During the second day of extinction, responding was reduced in control rats by $\sim 34$ and $\sim 22 \%$ for freezing and USV, respectively.

cause of obvious variance heterogeneity. The frequency distributions of level of freezing in the control and lesioned groups did not overlap. On the first day of testing, the highest level of freezing to the context in any ACe-lesioned animal was $14.0 \%$, whereas the lowest level in any sham-operated control rat was $25.3 \%$. There was limited overlap in the distributions of USV because some control rats failed to vocalize, as noted below.

During training, there were significant group differences in both post-shock freezing (Mann-Whitney rank sum test; $p<$ 0.001) and USV $(p<0.001)$ (cf. Goldstein et al., 1996). Both freezing and USV were almost completely abolished as CRs to the training context on both retention tests (Fig. 4), an effect that was statistically significant on both test days for both USV (MannWhitney rank sum test; $p<0.001)$ and freezing $(p<0.001)$ (cf. Antoniadis and McDonald, 2000). Among the control animals, responding was reduced on the second day of testing by $\sim 34$ and $\sim 22 \%$ for freezing and USV, respectively. This extinction effect was statistically significant for freezing $\left(t_{(15)}=3.14 ; p<0.01\right)$ but not for USV $\left(t_{(15)}=1.33 ; p=0.20\right)$.

\section{ACe lesion effects on post-ejaculatory USV and freezing}

Post-ejaculatory USV and freezing, measured before and after ACe lesions, appeared to be unaffected by ACe ablation. Figure 5 shows a typical time course for USV and freezing during the post-ejaculatory period in two representative rats before (Fig. $5 A$ ) and after (Fig. 5B) one of them received bilateral ACe lesions and the other underwent a sham operation. The dots represent USVs, and the accompanying bars show freezing. Both animals exhibited the usual pattern of post-ejaculatory vocalization and freezing before and after surgery. There was no evidence of obligatory coupling between these behaviors in the sense that there were instances of freezing in the absence of vocalization and occasions of vocalization while not remaining immobile. In contrast to the dramatic effect of ACe lesions on training- and testing-associated freezing and USV, which is obvious even in individual animals (Fig. 3), ACe lesions had no apparent effect on either behavior when elicited by ejaculation (Figs. 5, 6).

ANOVA applied to total duration of freezing in the group data (plotted in Fig. 6A) revealed no significant effect of surgical condition $\left(F_{(1,12)}=0.96 ; p=0.35\right)$ or session $\left(F_{(1,12)}=0.075 ; p=\right.$ $0.79)$, and the surgery $\times$ session interaction $\left(F_{(1,12)}=0.52 ; p=\right.$ 0.82 ) was also insignificant. There was also no significant effect of the lesion on USV, which was evaluated in three ways: total duration, number of calls, and mean call duration (Fig. $6 B-D$ ). For total USV duration (Fig. $6 B$ ), there was no significant effect of surgical condition $\left(F_{(1,12)}=2.13 ; p=0.17\right)$ or session $\left(F_{(1,12)}=\right.$ $0.022 ; p=0.88)$, nor a significant surgery $\times$ session interaction $\left(F_{(1,12)}=0.51 ; p=0.49\right)$. Similarly, for a number of USVs (Fig. $6 C)$, there was no effect of surgical condition $\left(F_{(1,12)}=1.70 ; p=\right.$ $0.22)$ or session $\left(F_{(1,12)}=0.0098 ; p=0.92\right)$, and the surgery $\times$ session interaction $\left(F_{(1,12)}=0.18 ; p=0.68\right)$ was insignificant. There was also no significant lesion effect on the average duration of individual calls (Fig. $6 D$ ) or surgical condition $\left(F_{(1,12)}=0.023\right.$; $p=0.88)$ or session $\left(F_{(1,12)}=0.011 ; p=0.92\right)$ and the surgery $\times$ session interaction was insignificant $\left(F_{(1,12)}=0.15 ; p=0.71\right)$.

\section{Statistical relationships among the behaviors}

Table 1 shows the correlations among these behaviors as a function of the eliciting condition. The symbols in Table 1 were shortened to a two-letter code, in which the first letter, F or V, indicates the type of behavior (freezing or vocalization) and the second letter, T, E, or R, indicates the eliciting condition (training, ejaculation, or retention testing). The first numerical entry in Table 1 is the Spearman $r$ for the combined groups, and the second is the Pearson $r$ for the sham-operated control group. Overall, the correlation matrix reveals that levels of freezing and USV covary strongly when these behaviors are elicited during training or retention testing but not when elicited by ejaculation.

In the combined data set, none of the nine Spearman correlations with ejaculation-elicited behaviors was statistically significant, but all of the remaining six Spearman correlations associated with training and retention testing-elicited behaviors were significant $(p<0.01)$. Freezing and USV were strongly correlated during both training $(r=0.71)$ and testing $(r=0.63)$. Freezing rank order during training accounted for $\sim 68 \%$ of the variance in freezing rank order during testing and $\sim 49 \%$ of the variance in USV rank order during testing. The overall pattern of correlations was similar in the subset of sham-operated control animals. None of the nine Pearson correlations with ejaculationelicited behaviors were significant, but five of the six remaining Pearson correlations with training- and retention-elicited behaviors were significant $(p<0.05)$. Among the control animals, freezing and USV were again strongly correlated during both training $(r=0.88)$ and testing $(r=0.82)$. Freezing during acquisition accounted for $58 \%$ of variance in freezing during retention testing and $35 \%$ of the variance in USV during retention testing.

The temporal relationships between the onsets of freezing and USV depended on whether these responses were elicited by the context or ejaculation. Figure 7 shows the mean \pm SE latency to the first USV call and to the first episode of freezing in response to the context and after ejaculation. Because some sham-operated control rats $(n=16)$ failed to vocalize (cf. Lee et al., 2000) during training ( 2 of 16 ) or retention testing ( 3 of 16), the values in 
Figure 7 reflect the mean latencies of those that did vocalize. The mean onset latency was significantly shorter for freezing than for USV during both training (paired $t$ test; $t_{(13)}$ $=2.24 ; p<0.05)$ and retention testing (paired $t$ test; $\left.t_{(12)}=2.77 ; p<0.05\right)($ Fig. $7 A$ ). After ejaculation, however, the mean onset latency was shorter for USV than for freezing (Fig. 7B), differences that were not statistically significant (paired $t$ test; $t_{(13)}=0.79$ and 0.35 , respectively, for preoperative and postoperative measures).

Ejaculation-produced USV commonly began in association with grooming and licking of lower body parts. By definition, the latter two behaviors precluded freezing in the beginning of the post-ejaculatory vocalization period. Freezing increased as the frequency of grooming and licking declined but was sometimes interrupted by changes of position, which could be initiated by an approaching female partner (cf. Barfield and Geyer, 1972). The different relative onset latencies seem to reflect that fact that ejaculation is associated with behaviors that are compatible with USV but not freezing, whereas the training context does not elicit these competing behaviors.

\section{Discussion}

The results are inconsistent with the motor-deficit hypothesis (Fig. 8A) for freezing. The reason that ACe-lesioned rats fail to exhibit freezing CRs clearly is not because they are physically unable to remain immobile, because freezing after ejaculation was entirely normal (Figs. 5, 6). The motor-deficit hypothesis (Fig. 8A) can neither account for this fact nor the fact, further discussed below, that levels of freezing and USV were positively correlated when elicited as CRs but uncorrelated when elicited as unconditional responses (URs) (Table 1). The ACe lesion effect was not limited to freezing as a CR. Context-elicited USV was also impaired (Figs. 3, 4). More generally, we know that the effect of ACe damage is not limited to persistent CRs, such as freezing and USV. Circumscribed damage to ACe also blocks conditional eyeblink facilitation (Choi et al., 2001), a CR that appears to peak near the expected time of the US (Lindquist and Brown, 2002).

\section{CR-deficit hypothesis}

A parsimonious alternative hypothesis is that the motor regions of the brainstem that are necessary to produce freezing and USV are independently accessible through parallel CS-CR and US-UR pathways (Fig. $8 \mathrm{~B}$ ). Interference with amygdalar function at the time of testing appears to block or impair all conventional Pavlovian CRs to aversive conditioning that have been carefully studied. In addition to the three CRs already mentioned, the list includes autonomic responses, analgesia, defecation, certain audible vocalizations, and startle facilitation (Kapp et al., 1979; Hitchcock and Davis, 1986; Iwata et al., 1986; Fanselow and Helmstetter, 1988; Lee et al., 2000; Fendt, 2001; Borszcz and Leaton, 2003). On the other hand, AM damage clearly does not prevent freezing and USV as URs to ejaculation (Figs. 5, 6). Two recent studies have shown that freezing is also spared as a UR in AM-lesioned rats when the US is a natural predator odor (Wal-
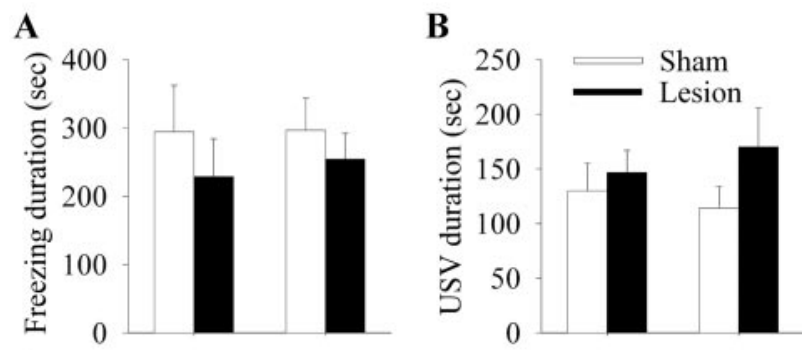

C

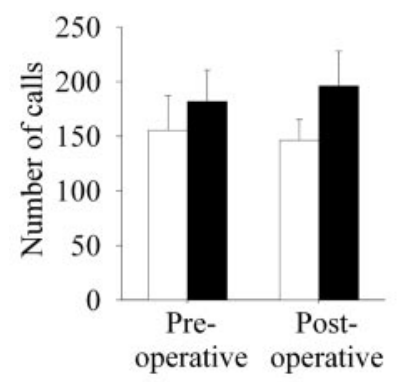

D

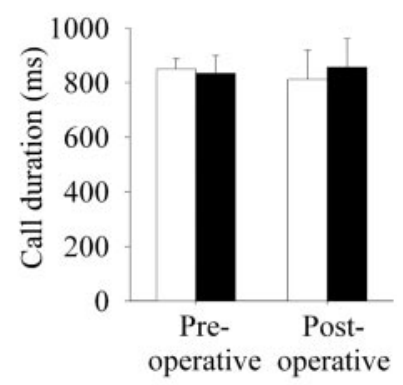

Figure 6. Preoperative and postoperative USV and freezing during the post-ejaculatory period in ACe-lesioned and sham-operated control rats. $A$, There were no significant differences between experimental and control animals in the mean duration of freezing. $B-D$, There were also no significant differences between experimental and control animals in regard to the mean USV duration $(B)$, the mean number of calls $(C)$, or the mean call duration $(D)$.

lace and Rosen, 2001; Fendt et al., 2003). These studies are worth repeating with lesions restricted to ACe.

\section{Parallel access to independent motor centers}

There was no evidence of obligatory coupling between the motor centers that control freezing and USV on the basis of the cooccurrence of these behaviors, their sequencing, or correlations between them. Under all experimental conditions, either behavior could occur in the absence of the other (Figs. 3, 5), and their relative onset latencies depended on the eliciting conditions (Fig. 
Table 1. Correlation matrix for freezing and USV elicited in three ways

\begin{tabular}{|c|c|c|c|c|c|c|}
\hline Variable & FR & VR & $\mathrm{FT}$ & VT & $\mathrm{FE}$ & VE \\
\hline FR & 1.0 & $0.63^{* *} / 0.82^{* *}$ & $0.89^{* *} / 0.76^{* *}$ & $0.64^{* *} / 0.60^{*}$ & $-0.29 / 0.49$ & $-0.17 / 0.27$ \\
\hline VR & & 1.0 & $0.64^{* *} / 0.59^{*}$ & $0.61^{* *} / 0.34$ & $-0.09 / 0.61$ & $-0.18 / 0.01$ \\
\hline $\mathrm{FT}$ & & & 1.0 & $0.71^{* *} / 0.88^{* *}$ & $-0.19 /-0.32$ & $-0.08 / 0.59$ \\
\hline VT & & & & 1.0 & $0.08 /-0.28$ & $0.32 / 0.75$ \\
\hline $\mathrm{FE}$ & & & & & 1.0 & $0.41 / 0.02$ \\
\hline VE & & & & & & 1.0 \\
\hline
\end{tabular}

The first entry is the Spearman $r$ for the entire data set, and the second entry is the Pearson $r$ for the sham-operated controls. F, V, Behavior measured (freezing or vocalization); R, T, E, eliciting condition (retention testing, training, or ejaculation). ${ }^{*} p<0.05 ;{ }^{* *} p<0.01$. The sample size is smaller for ejaculation-elicited freezing and USV ( $\left.n=14\right)$ than for conditioning-associated freezing and USV ( $\left.n=34\right)$.

A

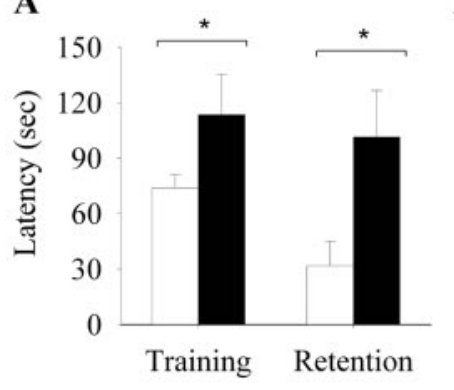

Conditioning
B

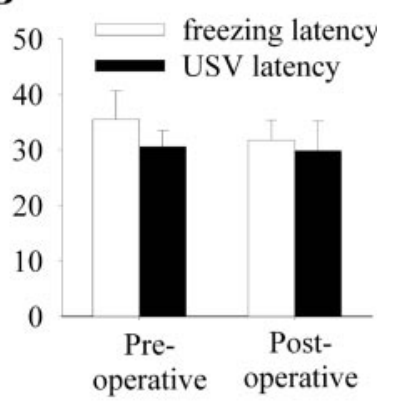

Ejaculation

Figure 7. Temporal relationships between USV and freezing as a function of the eliciting condition. $A$, Mean \pm SE latencies to the first USV (black bars) and to the first freezing episode (white bars) during training (left) and testing (right). Latency was measured during training from the time of the first shock US. During retention testing, latency was measured from the time the animal was introduced into the conditioning chamber. Data are from sham-operated rats (ACe-lesioned animals fail to vocalize and freeze during conditioning and testing). Asterisks denote that the mean freezing latency was significantly shorter than the mean USV latency during both training and retention testing $(p<0.001)$. B, Mean \pm SE latencies to the first USV and the first instance of freezing during the post-ejaculatory period. The post-ejaculatory latencies were measured from the time of last mounting. The mean latency order was reversed in this case, being shorter for USV than for freezing, but the differences between the means were not statistically significant. The mean onset latencies for USV and freezing were unaffected by ACe lesions.

7). When these responses were elicited by the context, and therefore ACe dependent, the mean onset latency was shorter for freezing than for USV (Fig. 7A). In contrast, when they were produced after ejaculation, and therefore ACe independent, the mean onset latency was shorter for USV than for freezing (Fig. 7B).

The six significant correlations among freezing and USV associated with conditioning and testing (Table 1) presumably reflect the fact that these behaviors are all triggered or controlled by ACe output that is elicited by the same context. The nine insignificant correlations (Table 1) suggest that, during the postejaculatory period, the motor centers for freezing (Bandler et al., 1985; Fanselow, 1991; Depaulis et al., 1992) and USV (Brudzynski and Bihari, 1990; Brudzynski, 1994; Brudzynski and Barnabi, 1996) are not both being driven by a common input but rather by parallel circuits, neither of which depends on ACe. Our working hypothesis (Fig. $8 \mathrm{~B}$ ) is that the motor centers responsible for freezing and USV can function independently of each other and can therefore have uncorrelated outputs unless they are separately controlled by correlated inputs or jointly driven by a common input, such as ACe, when the eliciting stimulus is a CS.

\section{Interpretation of responding during acquisition}

The CR-deficit hypothesis implies that freezing during training is primarily or entirely a $\mathrm{CR}$ to the conditioning context rather than a UR to the shock (Fig. 8 B). This interpretation was motivated by Fanselow's discovery that, when rats are shocked after being

A

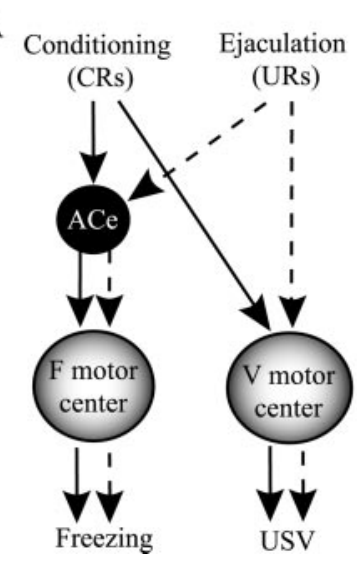

B

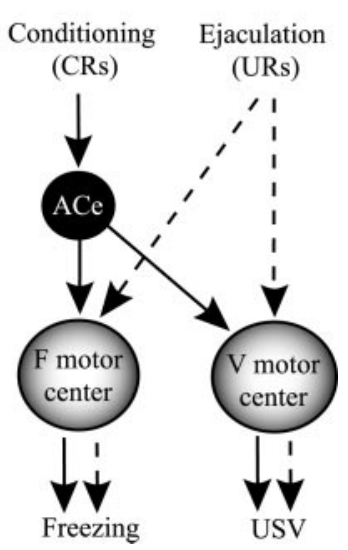

Figure 8. Two interpretations of ACe lesion effects. $A$, The rejected motor-deficit hypothesis, according to which ACe lesions should block freezing when elicited as a CR and UR but have no effect on USV, however elicited. $B$, The CR-deficit hypothesis, according to which ACe lesions should block both freezing and USV when elicited as CRs to aversive conditioning but have no effect on either behavior when elicited as URs to ejaculation. The core idea is that conventional Pavlovian CRs to aversive conditioning are all funneled through ACe to the relevant motor centers, which can be independently driven by some USs. Solid lines with arrows, CS-CR pathways; broken lines with arrows, US-UR pathways; $F$, freezing; V, vocalization.

placed in a novel context, they do not freeze unless the shock onset is sufficiently delayed to support context conditioning (Fanselow, 1980). The implication, that freezing during training represents the immediate expression of context conditioning rather than a UR to shock (Fanselow and Lester, 1988; Fanselow, 1989), suggests a simple explanation for the sizable shared variance in freezing during training and testing (Table 1 and associated discussion). Recall that $58-68 \%$ of the variance in freezing during retention testing was predicted by freezing during training.

The obvious explanation, based on Fanselow's results, is that the immediate expression of context conditioning is a good predictor of its expression $1 \mathrm{~d}$ later. The sizeable shared variance might not have been expected if, for example, post-shock freezing were a UR to the shock or a non-associative form of sensitization. If both post-shock freezing and USV are presumed to reflect context conditioning, then the CR latency data (Fig. $7 A$ ) imply that context conditioning tended to reach threshold for expressing both CRs within two US presentations (Fig. 7A). The reason is that the mean \pm SE latencies for training-produced freezing $(74 \pm 7 \mathrm{sec})$ and USV $(113 \pm 22 \mathrm{sec})$ (Fig. 7A, left) were both less than the latency of the third US presentation $(120 \mathrm{sec})$ (Fig. 3, column 1).

\section{Suggestions for additional analysis}

The present results immediately suggest three additional lines of research. The first concerns the use of electrolytic lesions, which were chosen to be consistent with the bulk of the research on ACe. 
Neurotoxic lesion studies (Jarrard, 1989) will be needed to determine whether the ACe lesion effects reported here and previously (for additional discussion, see Choi et al., 2001) result from eliminating neurons whose cell bodies lie within ACe, as we tentatively assume, on the basis of the functional neuroanatomy (LeDoux et al., 1988), or from damaging axons in ACe whose somata are located elsewhere. The present analysis is not inconsistent with the possibility that the observed lesion effects reflect damage to fibers of passage, as claimed in one preliminary report (Lee et al., 2002).

The second concerns our inference that post-shock USV, like post-shock freezing, is a CR to the context. Whereas Fanselow measured freezing but not USV (Fanselow, 1980), the circuit model (Fig. $8 \mathrm{~B}$ ) predicts that the results would be the same if USV were the CR. This expectation is not unreasonable, because freezing and USV tend to covary strongly as defensive CRs (Table 1) (Lee et al., 2000), but additional studies will be needed to test this prediction. Certain audible post-shock vocalizations are in fact URs that are organized by circuitry lower in the neuraxis than the amygdala (Borszcz and Leaton, 2003).

A third concerns the generality of the CR-deficit hypothesis. Although ACe lesions do appear to block or impair every relevant Pavlovian CR, we certainly cannot conclude that these lesions have no effect on any URs (Borszcz and Leaton, 2003). The limits of this hypothesis can be further explored by analyzing the effects of ACe lesions on additional behaviors that can be elicited both as CRs and URs. Conditional eye-blink facilitation (Lam et al., 1996) might serve this purpose. Whereas ACe lesions are known to eliminate facilitation of the eye-blink reflex as a CR to a cue (Choi et al., 2001), we do not know whether the same lesions also diminish eye-blink facilitation as a UR, for example, to a natural predator odor, a loud noise, the sight of a cat, or a distress signal from a conspecific (Blanchard et al., 1991, 2003; Brudzynski and Chiu, 1995; Powers et al., 1997; Brudzynski, 2001; Dielenberg and McGregor, 2001; Wallace and Rosen, 2001).

\section{Mnemonic functions of ACe}

Although normal ACe function clearly is required to express Pavlovian CRs to aversive conditioning, there is no compelling evidence regarding the exact nature of its role in CR production. One possibility is that ACe circuitry is necessary simply by virtue of being an essential part of the final common pathway for CR elicitation (LeDoux, 2000), as suggested by the circuit in Figure $8 B$. This "funnel hypothesis" furnishes a parsimonious explanation for the fact that ACe lesions blocked freezing and USV as CRs but not as URs and also the finding that these behaviors were strongly correlated when elicited as CRs but not when elicited as URs (Table 1).

However, there is reason to suspect that the role of ACe in Pavlovian fear conditioning is not limited to relaying neural CRs to the relevant motor centers (Borszcz and Leaton, 2003). Because ACe receives a direct US input from pain pathways (Gauriau and Bernard, 2002), it is conceivable that CS-US pairings can create neuronal modifications within ACe that are necessary or important for CR production. Furthermore, there is evidence that the output from the amygdala may control memory formation or consolidation in certain circuits to which the amygdala projects (Dalmaz et al., 1993; Choi et al., 2001), such as the basal ganglia (Robinson and Beart, 1988; Brog et al., 1993; McDonald, 2003). In this regard, it is worth noting that ACe itself is arguably a substructure of the basal ganglia (Swanson and Petrovich, 1998; McDonald, 2003).

\section{References}

Anderson JW (1954) The production of ultrasonic sounds by laboratory rats and other mammals. Science 119:808-809.

Antoniadis EA, McDonald RJ (1999) Discriminative fear conditioning to context expressed by multiple measures of fear in the rat. Behavioral Brain Res 101:1-13.

Antoniadis EA, McDonald RJ (2000) Amygdala, hippocampus and discriminative fear conditioning to context. Behavioral Brain Res 108:1-19.

Bandler R, Depaulis A, Vergnes M (1985) Identification of midbrain neurones mediating defensive behaviour in the rat by microinjections of excitatory amino acids. Behav Brain Res 15:107-119.

Barfield RJ (1975) The ultrasonic postejaculatory vocalization and the postejaculatory refractory period of the male rat. J Comp Physiol Psychol 88:723-734.

Barfield RJ, Geyer LA (1972) Sexual behavior: ultrasonic postejaculatory song of the male rat. Science 176:1349-1350.

Barrionuevo G, Brown TH (1983) Associative long-term synaptic potentiation in hippocampal slices. Proc Natl Acad Sci USA 80:7347-7351.

Blair HT, Schafe GE, Bauer EP, Rodrigues SM, LeDoux JE (2001) Synaptic plasticity in the lateral amygdala: a cellular hypothesis of fear conditioning. Learn Mem 8:229-242.

Blanchard DC, Blanchard RJ (1972) Innate and conditioned reactions to threat in rats with amygdaloid lesions. J Comp Physiol Psychol 81:281-290.

Blanchard DC, Markham C, Yang M, Hubbard D, Madarang E, Blanchard RJ (2003) Failure to produce conditioning with low-dose trimethylthiazoline or cat feces as unconditioned stimuli. Behav Neurosci 117:360-368.

Blanchard RJ, Blanchard DC (1969) Crouching as an index of fear. J Comp Physiol Psychol 67:370-375.

Blanchard RJ, Blanchard DC, Agullana R, Weiss S (1991) Twenty-two kHz alarm cries to presentation of a predator, by laboratory rats living in visible burrow system. Physiol Behav 50:967-972.

Blaustein JD, Wade GN (1977) Sequential inhibition of sexual behavior by progesterone in female rats: comparison with a synthetic antiestrogen. J Comp Physiol Psychol 91:752-760.

Borszcz GS, Leaton RN (2003) The effect of amygdala lesions on conditional and unconditional vocalizations in rats. Neurobiol Learn Mem 79:212-225.

Brog JS, Salyapongse A, Deutch AY, Zahm DS (1993) The patterns of afferent innervation of the core and shell in the "accumbens" part of the rat ventral striatum: immunohistochemical detection of retrogradely transported fluoro-gold. J Comp Neurol 338:255-278.

Brown TH, Lindquist DL (2003) Long-term potentiation: amygdala. In: Learning and memory (Byrne JH, Eichenbaum H, Roediger H, Thompson RF, eds), pp 342-343. Farmington Hills, MI: Macmillan Reference.

Brudzynski SM (1994) Ultrasonic vocalization induced by intracerebral carbachol in rats: localization and dose-response study. Behav Brain Res 63:133-143.

Brudzynski SM (2001) Pharmacological and behavioral characteristics of 22 $\mathrm{kHz}$ alarm calls in rats. Neurosci Biobehav Rev 25:611-617.

Brudzynski SM, Barnabi F (1993) Analysis of $22 \mathrm{kHz}$ ultrasonic vocalization in laboratory rats: long and short calls. Physiol Behav 54:215-221.

Brudzynski SM, Barnabi F (1996) Contribution of the ascending cholinergic pathways in the production of ultrasonic vocalization in the rat. Behav Brain Res 80:145-152.

Brudzynski SM, Bihari F (1990) Ultrasonic vocalization of rats produced by cholinergic stimulation of the brain. Neurosci Lett 109:222-226.

Brudzynski SM, Chiu EMC (1995) Behavioral response of laboratory rats to playback of $22 \mathrm{kHz}$ ultrasonic calls. Physiol Behav 57:1039-1044.

Cahill L, Weinberger NM, Roozendaal B, McGaugh JL (1999) Is the amygdala a locus of "conditioned fear"? Some questions and caveats. Neuron 23:227-228.

Cahill L, McGaugh JL, Weinberger NM (2001) The neurobiology of learning and memory: some reminders to remember. Trends Neurosci 24:578-581.

Canli T, Brown TH (1996) Amygdala stimulation enhances the rat eyeblink reflex through a short-latency mechanism. Behav Neurosci 110:51-59.

Choi J-S, Lindquist DL, Brown TH (2001) Amygdala lesions block conditioned enhancement of the early component of the rat eyeblink reflex. Behav Neurosci 115:764-775. 
Dalmaz C, Introini-Collison IB, McGaugh JL (1993) Noradrenergic and cholinergic interactions in the amygdala and the modulation of memory storage. Behav Brain Res 58:167-174.

Depaulis A, Keay KA, Bandler R (1992) Longitudinal neuronal organization of defensive reactions in the midbrain periaqueductal gray region of the rat. Exp Brain Res 90:307-318.

Dielenberg RA, McGregor IS (2001) Defensive behavior in rats towards predatory odors: a review. Neurosci Biobehav Rev 25:597-609.

Fanselow MS (1980) Conditional and unconditional components of postshock freezing. Pavlov J Biol Sci 15:177-182.

Fanselow MS (1989) The adaptive function of conditioned defensive behavior: an ecological approach to pavlovian stimulus substitution theory. In: Ethoexperimental approaches to the study of behavior (Blanchard RJ, Brain PF, Blanchard DC, Parmigiani S, eds), pp 151-166. Boston: Kluver Academic.

Fanselow MS (1991) The midbrain periacqueductal gray as a coordinator of action in response to fear and anxiety. In: The midbrain periacqueductal gray matter: functional, anatomical and immunohistochemical organization (Depaulis A, Bandler R, eds), pp 151-174. New York: Plenum.

Fanselow MS, Helmstetter FJ (1988) Conditioned analgesia, defensive freezing and bensodiazepines. Behav Neurosci 102:233-243.

Fanselow MS, LeDoux JE (1999) Why we think plasticity underlying Pavlovian fear conditioning occurs in the basolateral amygdala. Neuron 23:229-232.

Fanselow MS, Lester LS (1988) A functional behavioristic approach to aversively motivated behavior: predatory imminence as a determinant of the topography of defensive behavior. In: Evolution and learning (Bolles RC, Beecher MD, eds), pp 185-211. Hillsdale, NJ: Earlbaum.

Faulkner B, Tieu KH, Brown TH (1997) Mechanisms for temporal encoding in fear conditioning. In: Computational neuroscience (Bower, ed), pp 641-645. New York: Plenum.

Fendt M (2001) Injections of the NMDA receptor antagonist aminophosphonopentanoic acid into the lateral nucleus of the amygdala block the expression of fear-potentiated startle and freezing. J Neurosci 21:4111-4115.

Fendt M, Endres, Apfelbach (2003) Temporary inactivation of the bed nucleus of the stria terminalis but not of the amygdala blocks freezing induced by trimethylthiazoline, a component of fox feces. J Neurosci 23:23-28.

Gauriau C, Bernard JF (2002) Pain pathways and parabrachial circuits in the rat. Exp Physiol 87:251-258.

Goldstein LE, Rasmusson AM, Bunney BS, Roth RH (1996) Role of amygdala in the coordination of behavioral, neuroendocrine, and prefrontal cortical monoamine reasponses to psychological stress in the rat. J Neurosci 15:4787-4798.

Hitchcock JM, Davis M (1986) Lesions of the amygdala, but not of the cerebellum or red nucleus, block conditioned fear as measured with the potentiated startle paradigm. Behav Neurosci 100:11-22.

Iwata J, LeDoux JE, Reis DJ (1986) Destruction of intrinsic neurons in the lateral hypothalamus disrupts cardiovascular but not behavioral conditoned emotional responses. Brain Res 368:161-166.

Jarrard LE (1989) On the use of ibotenic acid to lesion selectively different components of the hippocampal formation. J Neurosci Methods 29:251-259.

Kapp BS, Frysinger RC, Gallagher M, Haselton J (1979) Amygdala central nucleus lesions: effects on heart rate conditioning in the rabbit. Physiol Behav 23:1109-1117.

Kelso SR, Brown TH (1986) Differential conditioning of associative synaptic enhancement in hippocampal brain slices. Science 232:85-87.

Kelso SR, Ganong AH, Brown TH (1986) Hebbian synapses in hippocampus. Proc Natl Acad Sci USA 83:5326-5330.
Kim JJ, Rison RA, Fanselow MS (1993) Effects of amygdala, hippocampus, and periaqueductal grey lesions on short- and long-term contextual fear. Behav Neurosci 107:1093-1098.

Lam Y, Wong A, Canli T, Brown TH (1996) Conditioned enhancement of the early component of the rat eyeblink reflex. Neurobiol Learn Mem 66:212-220.

LeDoux JE (2000) Emotion circuits in the brain. Annu Rev Neurosci 23:155-184.

LeDoux JE, Iwata P, Cicchetti P, Reis DJ (1988) Different projections of the central amygdaloid nucleus mediate autonomic and behavioral correlates of conditioned fear. J Neurosci 8:2517-2529.

LeDoux JE, Cicchetti P, Xagoraris A, Romanski LM (1990) The lateral amygdaloid nucleus: sensory interface of the amygdala in fear conditioning. J Neurosci 10:1062-1069.

Lee H, Kim JJ (1998) Amygdalar NMDA receptors are critical for new learning in previously fear-conditioned rats. J Neurosci 18:8444-8454.

Lee HJ, Choi J-S, Brown TH, Kim JJ (2000) Amygdala N-methyl-Daspartate (NMDA) receptors are critical in mediating the expression of multiple conditioned fear responses. J Neurosci 21:4116-4124.

Lee TK, Han JS, Lee HJ, Kim JJ (2002) Selective neurotoxin lesions of the basolateral and central amygdala differentially affect $22 \mathrm{kHz}$ ultrasonic vocalization of rats in fear conditioning. Soc Neurosci Abstr 28:379.372.

Levy WB, Steward O (1979) Synapses as associative memory elements in the hippocampal formation. Brain Res 175:233-245.

Lindquist DH, Brown TH (2002) Temporal encoding in fear conditioning revealed through modulation of the unconditioned eyeblink reflex. Soc Neurosci Abstr 28:85.84.

Maren S (1999) Long-term potentiation in the amygdala: a mechanism for emotional learning and memory. Trends Neurosci 22:561-567.

McDonald AJ (2003) Is there an amygdala and how far does it extend?: an anatomical perspective. In: The amygala in brain runction: basic and clinical approaches (Shinnick-Gallagher P, Pitkanen A, Shekhar A, Cahill L, eds), pp 1-21. New York: Annals of the New York Academy of Science.

McGann JP, Brown TH (2000) Fear conditioning model predicts key temporal aspects of conditioned response production. Psychobiology 28:303-313.

Paxinos G, Watson C (1998) The rat brain, Ed 4. San Diego: Academic Press.

Powers AS, Schicatano EJ, Basso MA, Evinger C (1997) To blink or not to blink: inhibition and facilitation of reflex blinks. Exp Brain Res 113:283-290.

Robinson TG, Beart PM (1988) Excitant amino acid projections from rat amygdala and thalamus to nucleus accumbens. Brain Res Bull 20:467-471.

Swanson LW, Petrovich GD (1998) What is the amygdala? Trends Neurosci 21:323-331.

Tieu KH, Keidel AL, McGann JP, Faulkner B, Brown TH (1999) Perirhinalamygdala circuit-level computational model of temporal encoding in fear conditioning. Psychobiology 27:1-25.

Van der Poel AM, Miczek KA (1991) Long ultrasonic calls in male rats following mating, defeat and aversive stimulation: frequency modulation and bout structure. Behavior 119:127-142.

Vazdarjanova A, McGaugh JL (1998) Basolateral amygdala is not critical for cognitive memory of contextual fear conditioning. Proc Natl Acad Sci USA 95:15003-15007.

Vazdarjanova A, Cahill L, McGaugh JL (2000) Disrupting basolateral amygdala function impairs unconditioned freezing and avoidance in rats. Eur J Neurosci 14:709-718.

Wallace KJ, Rosen JB (2001) Neurotoxic lesions of the lateral nucleus of the amygdala decrease conditioned fear but not unconditioned fear of a predator odor: comparison with elctrolytic lesions. J Neurosci 21: 3619-3627. 